\section{Análise de custo-efetividade do reúso de cateteres de cinecoronariografia sob a perspectiva de uma instituição pública no Município do Rio de Janeiro, Brasil}

\author{
Cost-effectiveness analysis on the reutilization \\ of coronary artery catheters in a public hospital in \\ Rio de Janeiro, Brazil
}

\begin{abstract}
Análisis del coste-efectividad en la reutilización de catéteres bajo la perspectiva de una institución pública en la ciudad de Río de Janeiro, Brasil
\end{abstract}

\begin{abstract}
The aim of this study was to compare the costeffectiveness ratio of new versus reprocessed coronary artery catheters in a Federal public hospital. This was an analytical decision-making model prepared to estimate the cost-effectiveness ratio between two strategies in the use of materials in coronary artery catheterization, with pyrogenic reaction as the clinical outcome. Costs were estimated using direct data collection in the respective catheterization services and expressed in Brazilian Reais (R\$), with 2012 as the reference year. The decision-making tree was constructed with the probabilities of pyrogenic reaction as described in a clinical trial. The cost per catheter for reuse was $R \$ 109.84$, as compared to $R \$ 283.43$ for a new catheter. The reutilization strategy proved to be cost cost-effective, and the incremental cost-effectiveness ratio indicated that $R \$ 13,561.75$ would be spent to avoid one case of pyrogenic reaction. The study identified reuse of coronary artery catheters as a lower cost strategy compared to the exclusive use of new catheters, thus potentially assisting decision-making by health administrators.
\end{abstract}

Cardiac Catheterization; Equipament Reuse; Cost-Effectiveness Evaluation; Health Technology Assessment
Bruna Medeiros Gonçalves de Veras ${ }^{1}$

Kátia Marie Simões e Senna ${ }^{1}$

Marcelo Goulart Correia 1

Marisa Silva Santos 1

\section{Resumo}

O objetivo foi comparar a relação de custo-efetividade entre o uso de cateteres cardíacos novos com cateteres reprocessados sob a perspectiva de uma instituição pública federal. Foi elaborado um modelo analítico de decisão elaborado para estimar a razão de custo-efetividade entre duas estratégias de utilização de materiais para cateterismo cardíaco utilizando, como desfecho clínico, a ocorrência de reação pirogênica. Os custos foram estimados por coleta direta nos setores envolvidos e valorados em Real ( $R \$$ ) para o ano de 2012. A árvore de decisão foi construída com as probabilidades de pirogenia descritas em estudo clínico. O custo para o reúso foi de $R \$$ 109,84 , e, para cateteres novos, de $R \$ 283,43$. A estratégia de reúso demonstrou ser custo-efetiva, e a razão de custo-efetividade incremental indicou que, para evitar um caso de pirogenia, serão gastos $R \$ 13.561,75$. O estudo aponta o reúso de cateteres como uma estratégia de menor custo comparada ao uso exclusivo de cateteres novos e pode contribuir para a tomada de decisão dos gestores.

Cateterismo Cardíaco; Reutilização de Equipamento; Avaliação de Custo-Efetividade; Avaliação de Tecnologias de Saúde 


\section{Introdução}

Nas últimas décadas, a mortalidade por doenças cardiovasculares vem caindo no Brasil. No entanto, essas doenças ainda são as principais causas de morte na população com mais de 60 anos 1. Dados de 2011 do Departamento de Informática do SUS (DATASUS. Informações de saúde: mortalidade Brasil. http://tabnet.data sus.gov.br/cgi/tabcgi.exe?sim/cnv/obt10uf.def, acessado em Jun/2013) demonstram ainda que as doenças do aparelho circulatório representam $28,6 \%$ de todas as mortes registradas na população em geral. Essas taxas elevadas ainda ocorrem, possivelmente, pela alta prevalência e pelo baixo controle dos fatores de risco, como a hipertensão arterial 1 .

Em centros de referência para atendimento cardiológico, os procedimentos de hemodinâmica são fundamentais para o diagnóstico e a terapêutica de doenças cardiovasculares, mas requerem tecnologia e produtos de alto custo 2 .

A introdução de novas tecnologias nos sistemas de saúde tem gerado aumento dos gastos nos serviços de saúde. Desse modo, é cada vez mais comum a adoção de práticas e medidas que reduzam os gastos. Nesse contexto, a reutilização de produtos médico-hospitalares denominados como de uso único vem sendo adotada em várias instituições 3 .

Dentre os procedimentos hemodinâmicos mais realizados, destaca-se o exame de diagnóstico para as doenças coronarianas denominado cateterismo cardíaco ou cinecoronariografia. A demanda por esse procedimento nos países ocidentais é estimada em 6 mil procedimentos por milhão de habitantes ao ano ${ }^{4}$. Segundo Cardoso et al. 5, de 1.681 .584 procedimentos registrados entre 1998 a 2005 na Sociedade Latino-Americana de Cardiologia Intervencionista (SOLACI), $74,9 \%$ eram cateterismos diagnósticos.

Com a elevada demanda por esses procedimentos e o custo elevado como um dos seus fatores limitantes, reprocessar e reutilizar cateteres cardíacos são ações comuns ao redor do mundo 6 . Suécia, Alemanha, Estados Unidos e Canadá são exemplos de países que permitem o reprocessamento em hospitais e empresas terceirizadas desde que sejam respeitadas as regulamentações que requerem um processo semelhante ao de fabricação. Já nos países que possuem poucos recursos, como os pertencentes a África, Ásia, Europa Oriental, América do Sul e América Central, essa prática se mantém permitida, aguardando regulamentação específica 7 .

No Brasil, a regulamentação estabelecida pela Agência Nacional de Vigilância Sanitária (ANVISA) buscou inicialmente a uniformização dos processos como forma de garantir a qualidade do reprocessamento. Nesse sentido, foram publicadas duas Resoluções Especiais (RE). A RE no 2.605/2006 8 listou diversos produtos médicos considerados de difícil reprocessamento. Tais produtos foram determinados exclusivamente como de uso único, e o seu reprocessamento foi proibido. Na $R E$ no $2.606 / 2006^{9}$, são descritas as diretrizes para a elaboração e validação de protocolos de reprocessamento.

No que se refere à ocorrência de reações adversas em consequência do reúso de cateteres cardíacos para procedimentos diagnósticos, a literatura disponível nos apresenta resultados em que o risco de reação pirogênica é similar aos encontrados em cateteres novos 10,11,12,13, e que as possíveis alterações na integridade física desses podem ser suprimidas ao se respeitar protocolos validados que controlem o número de reprocessamentos, considerando o comportamento mecânico do material como prevenção de possíveis fraturas 14 .

Diante das evidências de baixo risco para eventos adversos associados ao reprocessamento de cateteres cardíacos demonstradas nos estudos analisados, dos elevados custos para a realização de procedimentos hemodinâmicos e compreendendo a importância do serviço de hemodinâmica para um centro de referência em cardiologia, o presente estudo objetivou analisar e comparar os custos da aquisição de novos materiais de cateterismo cardíaco com os custos do seu reprocessamento a fim de fornecer subsídios para o processo decisório de uma instituição pública federal no Município do Rio de Janeiro.

\section{Metodologia}

A análise deste estudo foi conduzida sob a perspectiva de uma instituição pública federal, de nível terciário, localizada no Município do Rio de Janeiro e integrante do Sistema Único de Saúde (SUS). Essa instituição possui profissionais que trabalham tanto em regime estatutário como celetista, além de pessoal terceirizado. Nesse cenário, a estimação dos custos de mão de obra tornou-se complexa, sendo paga por outra fonte não orçamentária, na medida em que os funcionários são remunerados por regime de contratação e suas respectivas cargas horárias.

O reprocessamento dos materiais de cateterismo cardíaco se inicia na instituição, em um setor denominado Central de Esterilização Externa (CEE), com a etapa de pré-lavagem e o acondicionamento dos materiais até que sejam encaminhados para a empresa terceirizada de esterilização por óxido de etileno. 
Esse setor foi criado pela instituição analisada com o objetivo de atender as recomendações da legislação brasileira ( $R E$ no 2.606/2006 da ANVISA) 9 e da literatura 7 .

O acompanhamento e a cronometragem da pré-lavagem de materiais resultantes de quatro procedimentos de cateterismo em adultos foram feitos em uma única observação, pois os procedimentos são padronizados. As demais informações para o levantamento dos custos foram obtidas a partir de questionamentos direcionados aos funcionários dos setores envolvidos.

Etapas do processo realizado na CEE: (1) recolhimento dos cateteres no setor de hemodinâmica; (2) paramentação (equipamentos de proteção individual); (3) lavagem dos cateteres; (4) irrigação dos cateteres com solução desincrostante; (5) enxágue dos cateteres com jatos de água e (6) secagem com ar comprimido.

Ao retornarem da empresa de esterilização externa, os cateteres são checados e redistribuídos ao setor de hemodinâmica.

Utilizando o software TreeAge Pro 2011 (TreeAge Software Inc., Williamstown, Estados Unidos), foi desenvolvido um modelo de decisão para estimar a razão de custo-efetividade entre duas estratégias de utilização de materiais para cateterismo cardíaco: reúso de materiais (estratégia 1) e aquisição de materiais novos (estratégia 2). O desfecho clínico considerado no modelo foi a ocorrência de reações pirogênicas. Tal escolha foi motivada pelos registros de surtos ocorridos na instituição estudada. Outros desfechos, como a possibilidade de fragmentação do cateter reprocessado 7,14 e de bacteremia 7,11 por contaminação do cateter, foram considerados eventos raros com o reprocessamento utilizando óxido de etileno, pela revisão da literatura e pela experiência da instituição. O pressuposto para o modelo de custo-efetividade foi de que um cateter reprocessado conforme o protocolo não fragmenta e não acarreta bacteremia.

Para a análise de custo relativa a cada estratégia, foi definido, como unidade de custo por procedimento, um conjunto (kit) básico de materiais utilizados no procedimento de cateterismo cardíaco, composto por: introdutor, cateter diagnóstico e cateter guia.

Os itens de custo considerados para cada estratégia são descritos conforme a Tabela 1 . Os respectivos valores monetários referentes ao ano de 2012, de acordo com os processos licitatórios da instituição estudada, são apresentados na Tabela 2.

Em relação ao kit básico de materiais, foi considerado o valor de aquisição de $\mathrm{R} \$ 46,00$ do cateter diagnóstico, que é específico para o procedimento estudado. Devido à diversidade de ti- pos e custos de compra de introdutores e guias, foi estabelecido um custo médio para cada item. São utilizados, na instituição, seis tipos de introdutor com preços que variam de $\mathrm{R} \$ 29,00$ a $\mathrm{R} \$$ 313,00 e cinco tipos de guia com preços variando de $\mathrm{R} \$ 58,00$ a $\mathrm{R} \$ 180,00$. As variações nos preços de aquisição desses itens foram consideradas na análise de sensibilidade. Os custos de água e luz considerados para a estratégia 1 (reúso) correspondem aos valores praticados pelas distribuidoras do Município do Rio de Janeiro em janeiro de 2012 .

As probabilidades de ocorrência de reações pirogênicas consideradas em cada estratégia basearam-se no estudo de Frank et al. 11, que demonstrou uma probabilidade de 0,044 $(4,4 \%)$ com o uso de cateteres novos e 0,06 (6\%) no caso dos cateteres reprocessados múltiplas vezes.

O número de reprocessamentos/cateter considerado no modelo seguiu as recomendações de Lucas et al. ${ }^{14}$, que demonstrou modificações nas propriedades dos polímeros dos cateteres a partir do quarto reprocessamento. Tais modificações consistem em rugosidades e microfissuras que poderiam propiciar o acúmulo de biofilme $\mathrm{e}$ microrganismos, além de contribuírem com possíveis fraturas dos materiais.

Na estratégia 1 (reúso), o custo final e a probabilidade de ocorrência de reações pirogênicas precisaram ser ajustados considerando que um mesmo cateter seria utilizado cinco vezes. A probabilidade de pirogenia foi de 0,057 , isto é, $1 / 5$ da probabilidade de ocorrência de pirogenia com o uso de cateteres novos, somado a $4 / 5$ da probabilidade de ocorrência de pirogenia com o uso de cateteres reprocessados. O custo do reúso correspondeu a $1 / 5$ do custo de um cateter novo e a $4 / 5$ do custo de um cateter reprocessado.

A análise de sensibilidade foi realizada a partir de plausibilidade clínica e busca bibliográfica das variações dos valores analisados na árvore de decisão, e, então, foi desenvolvido um diagrama de tornado para a verificação da influência das variáveis no resultado final.

A variação no custo do tratamento da pirogenia na análise de sensibilidade utilizou, como referência, o custo da internação por complicações de procedimentos cirúrgicos ou clínicos, conforme o SIGTAP (Ministério da Saúde. Sistema de Gerenciamento da Tabela de Procedimentos, Medicamentos e OPM do SUS. http://sigtap. datasus.gov.br/tabela-unificada/app/sec/inicio. jspSIGTAP, acessado em Jun/2013). 
Descrição do cálculo dos custos dos kits de cateterismo de uso único e reprocessados.

\begin{tabular}{|c|c|c|}
\hline & Definição & Fonte \\
\hline \multicolumn{3}{|l|}{ Kits novos } \\
\hline Custo de compra & $\begin{array}{l}\text { Valor pelo qual os materiais que compõem o kit foram } \\
\text { adquiridos pela instituição estudada }\end{array}$ & Almoxarifado da instituição \\
\hline Custo de estocagem & $\begin{array}{l}\text { Área }\left(\mathrm{m}^{2}\right) \text { ocupada por cada unidade que compõe o kit } \\
\text { multiplicada pelo custo por } 1 \mathrm{~m}^{2} \text { da estocagem externa }\end{array}$ & $\begin{array}{l}\text { - Custo/m2: almoxarifado da instituição } \\
\text { - Área ocupada pela unidade e dimensões do } \\
\text { produto: almoxarifado e sites de revendedores } \\
\text { na Internet }\end{array}$ \\
\hline Custo de transporte & $\begin{array}{l}\text { Volume }\left(\mathrm{m}^{3}\right) \text { ocupado por cada unidade que compõe o } \\
\text { kit multiplicado pelo custo por } 1 \mathrm{~m}^{3} \text { do transporte }\end{array}$ & $\begin{array}{l}\text { - Custo } \mathrm{m}^{3} \text { : almoxarifado da instituição } \\
\text { - Volume ocupado pela unidade e dimensões } \\
\text { dos produtos: almoxarifado e sites de } \\
\text { revendedores na Internet }\end{array}$ \\
\hline Custo de descarte & $\begin{array}{l}\text { Peso do kit a ser descartado multiplicado } \\
\text { pelo custo de } 1 \mathrm{~kg} \text { descartado }\end{array}$ & $\begin{array}{l}\text { - Custo kg: gerência de resíduos da instituição } \\
\text { - Peso de cada kit: pesagem dos materiais e } \\
\text { peso descrito em sites de revendedores na } \\
\text { Internet }\end{array}$ \\
\hline Custo total/unidade & Soma dos custos acima & \\
\hline \multicolumn{3}{|l|}{ Kits reprocessados } \\
\hline Custos gerais & $\begin{array}{l}\text { Custos para mais de um procedimento de hemodinâmica, } \\
\text { como, por exemplo: custos de paramentação dos } \\
\text { profissionais da Central de Esterilização Externa (CEE). } \\
\text { Os profissionais se paramentam uma única vez para o } \\
\text { reprocessamento de diversos materiais. Esses custos } \\
\text { foram somados e divididos pela quantidade total de kits } \\
\text { a serem reprocessados }\end{array}$ & $\begin{array}{l}\text { - Valor de compra dos itens de paramentação: } \\
\text { almoxarifado da instituição } \\
\text { - Valor da embalagem de solução } \\
\text { desincrostante: farmácia da instituição }\end{array}$ \\
\hline Custo do reprocessamento externo & $\begin{array}{c}\text { Valor cobrado por unidade reprocessada } \\
\text { pela firma terceirizada }\end{array}$ & $\begin{array}{l}\text { Nota fiscal da firma terceirizada (julho e } \\
\text { setembro de 2012): administração da instituição }\end{array}$ \\
\hline Custo de luz & $\begin{array}{l}\text { Média de tempo gasto para separação e relação de cada } \\
\text { kit na CEE multiplicadas pelo custo de luz na CEE por } \\
\text { minuto. Inclui também o tempo gasto na secagem, pois o } \\
\text { custo de ar comprimido está embutido no custo da luz }\end{array}$ & $\begin{array}{l}\text { Conta de luz (janeiro de 2012): setor financeiro } \\
\text { da instituição }\end{array}$ \\
\hline Custo de água & $\begin{array}{l}\text { Volume de água gasto na lavagem e no enxágue de } \\
\text { um kit. O volume é calculado multiplicando o tempo } \\
\text { gasto em cada uma dessas etapas pela vazão de } \\
\text { água por minuto. O custo é obtido multiplicando-se o } \\
\text { volume gasto pelo valor do litro de água cobrado pela } \\
\text { companhia fornecedora de água }\end{array}$ & $\begin{array}{l}\text { - Vazão de água: 5.875L/min (Psaltikidis et al. 3). } \\
\text { - Conta de água (janeiro/2012): setor financeiro } \\
\text { da instituição }\end{array}$ \\
\hline Paramentação & $\begin{array}{l}\text { Custos dos itens utilizados pelos profissionais para } \\
\text { proteção individual (touca, óculos, máscara, luva de } \\
\text { procedimento, capote) dividido pela média do número } \\
\text { de kits avaliados por paramentação }\end{array}$ & $\begin{array}{l}\text { - Custos dos itens: almoxarifado } \\
\text { - Média do número de itens: acompanhamento } \\
\text { na CEE da avaliação de dois grupos de materiais }\end{array}$ \\
\hline
\end{tabular}

\section{Resultados}

A árvore de decisão com as probabilidades e os valores encontrados para cada estratégia é apresentada na Figura 1. A estratégia de reúso demonstrou ser mais custo-efetiva comparada à estratégia de aquisição de materiais novos.

Ao considerar a pirogenia como desfecho de interesse, observou-se que a estratégia de reúso dos kits custa 2,5 vezes menos que a estratégia de kits novos (R\$ 109,84 e R\$283,43, respectivamente).

Os resultados de efetividade e custo do uso de kits novos versus reutilizados estão apresentados na Tabela 3. A razão de custo-efetividade incremental indica que, para evitar um caso de pirogenia, são gastos R\$ 13.561,75. 
Itens considerados na análise de custos.

\begin{tabular}{|c|c|}
\hline & Valor \\
\hline Kit de cateterismo novo & $\mathrm{R} \$ 279,82$ \\
\hline Valor de compra do kit & $\mathrm{R} \$ 277,33$ \\
\hline Introdutor $5,6,7$ ou 8 French & $\mathrm{R} \$ 114,33$ \\
\hline Guia & $\mathrm{R} \$ 117,00$ \\
\hline Cateter diagnóstico & $\mathrm{R} \$ 46,00$ \\
\hline Custo de estocagem do kit & $\mathrm{R} \$ 2,34$ \\
\hline Custo $/ \mathrm{m}^{2}$ de estocagem externa & $\mathrm{R} \$ 14,61$ \\
\hline Área total $\left(\mathrm{m}^{2}\right)$ ocupada por um kit & 0,16 \\
\hline Custo de transporte do kit & $R \$ 0,10$ \\
\hline Custo do transporte/m³ & $\mathrm{R} \$ 18,03$ \\
\hline Volume total $\left(\mathrm{m}^{3}\right)$ ocupado por um kit & 0,01 \\
\hline Descarte do kit & $R \$ 0,04$ \\
\hline Valor pago por kg & $\mathrm{R} \$ 0,42$ \\
\hline Pesos de um kit (kg) & 0,1 \\
\hline Kit de cateterismo reutilizado & $\mathrm{R} \$ 105,18$ \\
\hline Luz & $R \$ 4,38$ \\
\hline Custo de luz/min da CEE & $\mathrm{R} \$ 0,31$ \\
\hline Duração total do processo $(\mathrm{min}) /$ kit & 13,96 \\
\hline Paramentação & $\mathrm{R} \$ 10,18$ \\
\hline Tempo de duração da etapa (min) & 1,1 \\
\hline Número de kits reprocessados/paramentação & 4 \\
\hline Número de funcionários paramentados & 3 \\
\hline Capote & $R \$ 9,00$ \\
\hline Máscara & $\mathrm{R} \$ 0,80$ \\
\hline Óculos & $\mathrm{R} \$ 2,85$ \\
\hline Touca & $\mathrm{R} \$ 0,80$ \\
\hline Luva de procedimento & $\mathrm{R} \$ 0,12$ \\
\hline Etapa de recolhimento dos kits na hemodinâmica & $R \$ 0,56$ \\
\hline Tempo de duração da etapa (min) & 11,13 \\
\hline Número de kits reprocessados/etapa & 4 \\
\hline Custo de água para lavagem do contêiner & $\mathrm{R} \$ 0,51$ \\
\hline Custo da solução desincrostante & $\mathrm{R} \$ 1,73$ \\
\hline Custo de água para dois contêineres & $\mathrm{R} \$ 0,02$ \\
\hline Etapa de preparação para lavagem & $\mathrm{R} \$ 1,77$ \\
\hline Tempo de duração da etapa (min) & 6,37 \\
\hline Número de kits reprocessados/etapa & 4 \\
\hline Higienização das mãos (10mL de álcool gel) & $R \$ 0,06$ \\
\hline Campo cirúrgico & $\mathrm{R} \$ 4,99$ \\
\hline Luva estéril & $R \$ 0,62$ \\
\hline Seringa de $10 \mathrm{~mL}$ & $\mathrm{R} \$ 0,15$ \\
\hline Esponja & $\mathrm{R} \$ 1,24$ \\
\hline Etapa de lavagem & $R \$ 1,22$ \\
\hline Tempo de duração da etapa (min) & 3,24 \\
\hline Custo final da lavagem de um cateter & $\mathrm{R} \$ 1,14$ \\
\hline Custo final da lavagem por guia & $R \$ 0,02$ \\
\hline Custo final da lavagem da capa da guia & $\mathrm{R} \$ 0,03$ \\
\hline Custo final da lavagem do kit introdutor & $\mathrm{R} \$ 0,03$ \\
\hline
\end{tabular}

(continua) 


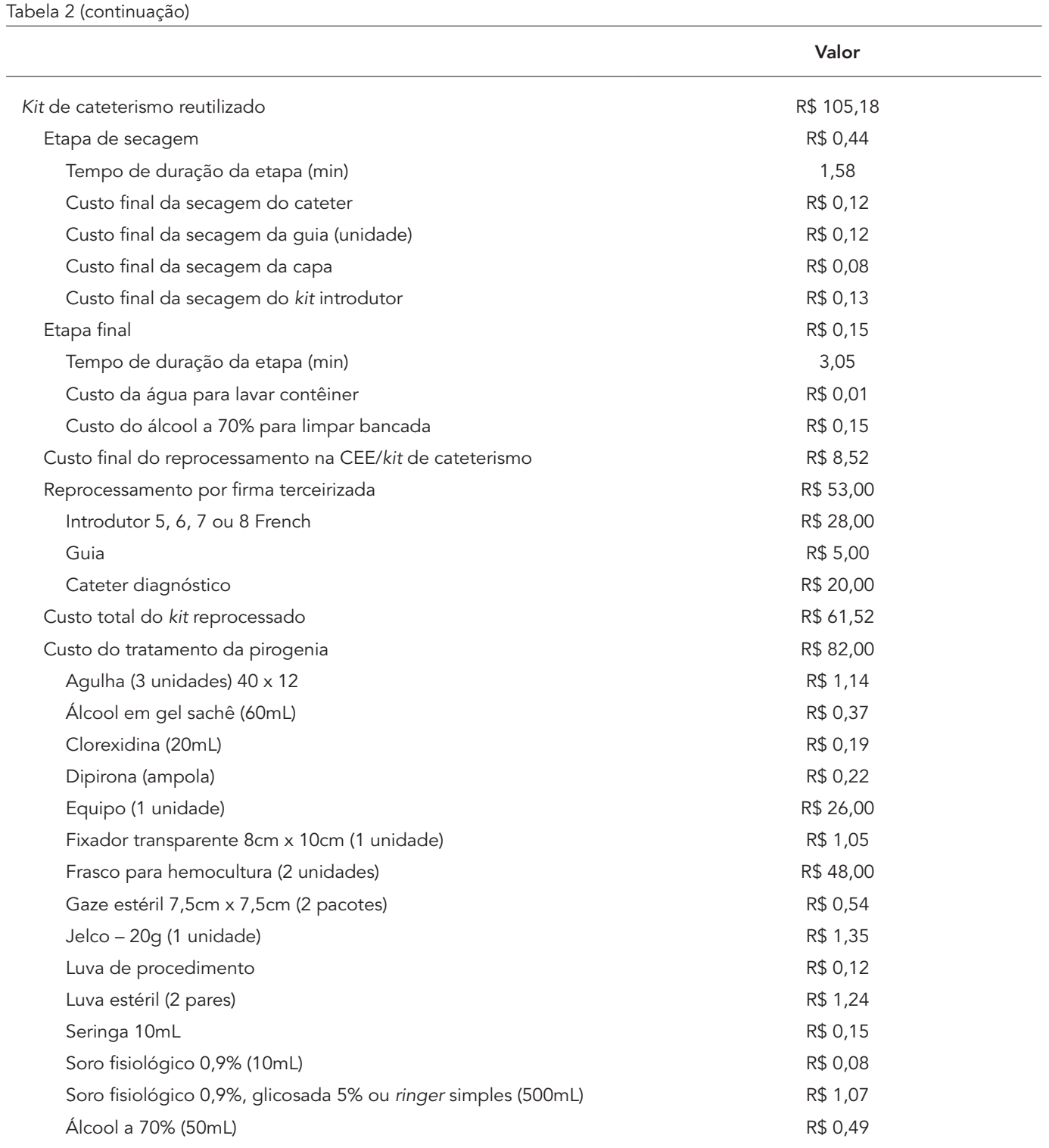

Para que as estratégias 1 e 2 tivessem o mesmo valor esperado, os custos de compra do kit deveriam ser reduzidos em $78,24 \%$.

O diagrama de tornado (Figura 2), gerado pela análise de sensibilidade, demonstrou que a variável que mais impactou na razão de custoefetividade incremental foi a probabilidade de pirogenia nos cateteres reprocessados, e as de menor impacto foram o custo do tratamento da pirogenia e o número de reprocessamentos.

\section{Discussão}

De acordo com Duffy et al. 6 , a prática de reprocessamento de cateteres de hemodinâmica ainda é controversa, pois existem diversos relatos que relacionam o reúso de cateteres cardíacos com um aumento do risco de exposição às endotoxinas que causam reações pirogênicas (calafrios, febre e hipotensão) ${ }^{15}$. Por outro lado, há outros estudos que sugerem que, se a limpeza e a esterilização dos cateteres cardíacos forem adequadamente realizadas, os cateteres podem ser reutilizados sem elevar os riscos dessas reações pirogênicas 6 .

Segundo Ribeiro 7, as preocupações com a segurança no reúso de artigos médicos de uso único estão relacionadas com a eficácia de sua limpeza e esterilização, com a integridade física e mecânica dos artigos e também com a segurança dos profissionais de saúde que reprocessam esses artigos. Bomfim et al. 16 confirmam que a etapa da limpeza é primordial para extinguir 


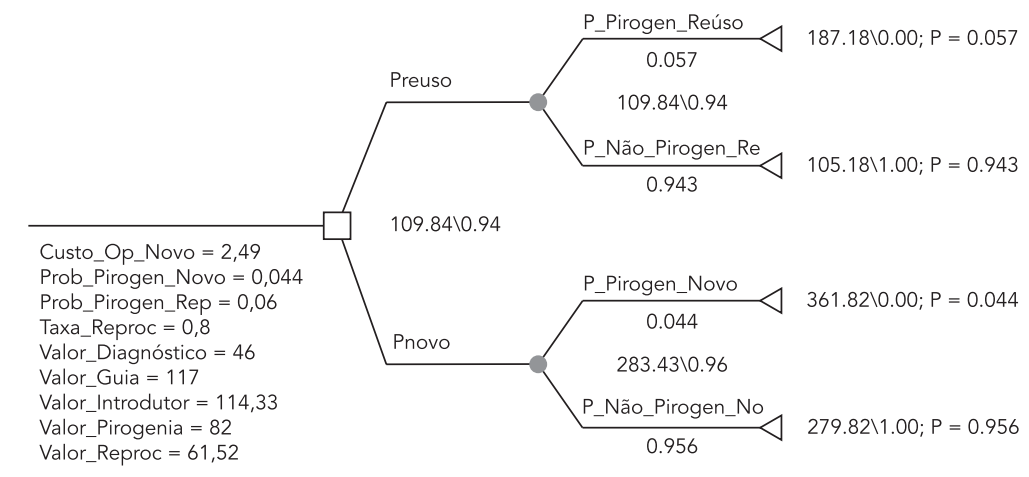

Custo_Op_Novo: custo operacional da utilização de um kit novo (compra, estocagem, transporte e descarte);

Prob_Pirogen_Novo: probabilidade de ocorrência de pirogenia com o uso de kits novos; Prob_Pirogen_Rep: probabilidade de ocorrência de pirogenia com o uso de kits reprocessados; Taxa_Reproc: taxa de reprocessamento por kit (4 reprocessamentos em 5 usos); Valor_Diagnóstico: custo de compra de um cateter diagnóstico novo; Valor_Guia: custo médio de compra de um cateter guia novo; Valor_Introdutor: custo médio de compra de um introdutor novo; Valor_Pirogenia: custo em reais do tratamento de um caso de pirogenia; Valor_Reproc: custo em reais de um reprocessamento de um kit; Preuso: probabilidade de uso de kit reutilizado para o cateterismo cardíaco; Pnovo: probabilidade de uso de kit novo para o cateterismo cardíaco; P_Pirogen_Reúso: probabilidade de ocorrência de pirogenia com o uso de kits reprocessados; P_Não_Pirogen_Re: probabilidade de não ocorrência de pirogenia com o uso de kits reprocessados; P_Não_Pirogen_No: probabilidade de não ocorrência de pirogenia com o uso de kits novos.

Tabela 3

Resultado da análise de custo-efetividade do uso de kits novos versus reutilizados.

\begin{tabular}{lccr}
\hline & Novo & Reúso & Custo incremental \\
\hline Custos & $\mathrm{R} \$ 283,428$ & $\mathrm{R} \$ 109,8376$ & $\mathrm{R} \$ 173,5904$ \\
\hline & Novo & Reúso & Efetividade incremental \\
\hline $\begin{array}{l}\text { Efetividade } \\
\text { Relação de custo-efetividade incremental }\end{array}$ & 0,956 & 0,9432 & 0,0128 \\
\hline
\end{tabular}

resíduos proteicos que favorecem o crescimento de biofilmes no lúmen do cateter. Diversos autores demonstram, em seus estudos, a importância de uma limpeza adequada dos cateteres ao registrarem surtos de pirogenia relacionados ao processo de limpeza e esterilização dos cateteres $6,17,18,19,20$.

Na literatura, verificou-se a predominância dos estudos envolvendo cateteres de angioplastia, com a identificação de alterações na sua integridade física (fendas, depressões, arranhões e complacência do balão) 21,22,23, bem como os que avaliaram os possíveis eventos clínicos ocasionados pelo reúso desses cateteres 22,24,25,26. Acredita-se que grande parte dos estudos com os cateteres de angioplastia tenham ocorrido pela dificuldade encontrada para sua limpeza e, consequentemente, reprocessamento, causada pela presença de um balão (ponto cego) em sua extremidade. Esses cateteres são descartados, após único uso, na instituição em respeito à legislação brasileira 8,9 que proíbe o reprocessamento de cateteres com fundo cego. 
Diagrama de tornado gerado pela análise de sensibilidade.

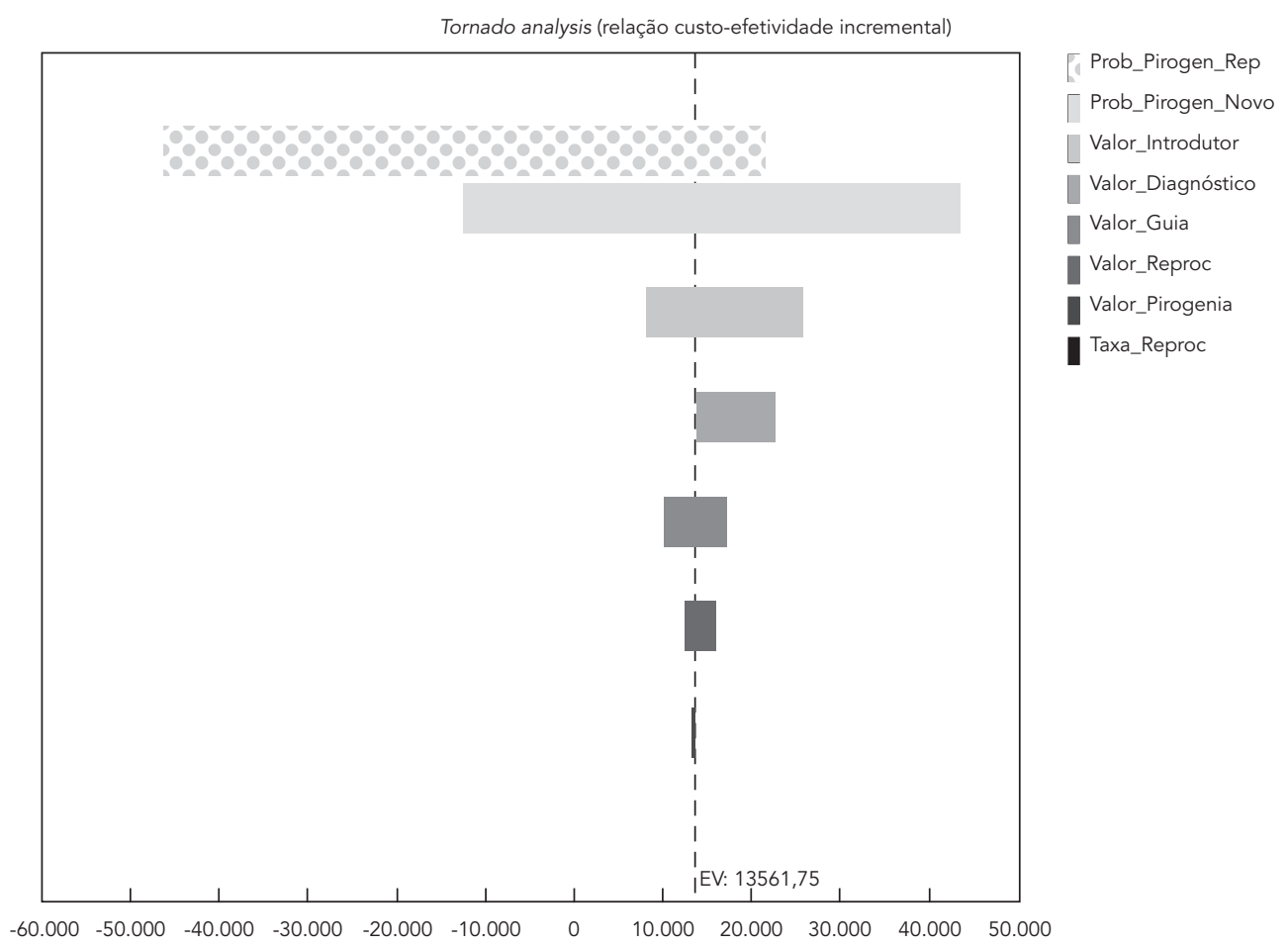

Prob_Pirogen_Rep: probabilidade de ocorrência de pirogenia com o uso de kits reprocessados; Prob_Pirogen_Novo: probabilidade de ocorrência de pirogenia com o uso de kits novos; Valor_Introdutor: custo médio de compra de um introdutor; Valor_Diagnóstico: custo de compra de um cateter diagnóstico; Valor_Guia: custo médio de compra de um cateter guia novo; Valor_Reproc: custo em reais de um reprocessamento de um kit; Valor_Pirogenia: custo em reais do tratamento de um caso de pirogenia; Taxa_Reproc: taxa de reprocessamento por kit; EV: razão de custo-efetividade incremental.

Segundo Dunn (2002, apud Bomfim et al. 16), alguns procedimentos em cardiologia intervencionista seriam comercialmente inviáveis sem o reprocessamento de materiais. A economia pode chegar a $50 \%$ quando o reprocessamento é realizado por empresas terceirizadas.

No presente estudo, a estratégia de reúso se mostrou custo-efetiva em relação à compra de cateteres novos. Todavia, é importante comentar que, caso a estratégia de compra de cateteres novos se mostrasse mais custo-efetiva, seria necessário um novo planejamento para atender à demanda da instituição, reprogramando as entregas, que deveriam ocorrer diversas vezes ao ano, de forma a não comprometer a realização dos procedimentos e considerando a diminuição dos custos com a estocagem externa. O processo de compra em uma instituição pública pode durar aproximadamente seis meses, por envolver diversas áreas, além de cumprir com todas as exigências da legislação pertinente ao uso correto do dinheiro público.

O modelo proposto não considerou alguns outros desfechos, como bacteremia, infecção e fratura dos cateteres. As pirogenias são as reações que encontram relatos na instituição estudada e que também estão descritas por outros autores 6,17,18,19,20.

Um surto de reações pirogênicas ocorrido durante o mês de janeiro de 2012, na instituição (comunicação verbal), registrou nove pacientes que apresentaram tremores, calafrios e/ou febre após cateterismo cardíaco diagnóstico, sem repercussão hemodinâmica. O surto foi resolvido com a readequação do protocolo de coleta e pré-lavagem dos cateteres antes do envio para a esterilização em óxido de etileno na empresa terceirizada. 
Não foram encontrados relatórios de agências reguladoras ou artigos que atribuíssem bacteremia especificamente ao reúso dos cateteres diagnósticos. Relatórios do Food and Drug Administration dos Estados Unidos (FDA) 27, referentes a dezembro de 2005 até julho de 2006, que incluíram 65 relatos de envolvimento de materiais de uso único reprocessados em eventos adversos, encontraram que as reações que envolviam os materiais reprocessados eram semelhantes às que envolviam materiais novos ou não reprocessados. Mais de 50 participantes de uma rede de 350 hospitais norte-americanos, com treinamento específico para identificar eventos adversos relacionados aos materiais médicos, pontuaram que, quando uma infecção ocorre, é difícil definir se a causa está relacionada ao reprocessamento de materiais de uso único. Os participantes também não relataram preocupações maiores com problemas mecânicos associados com materiais de uso único reprocessados, se comparados aos não reprocessados 27 .

O estabelecimento de protocolos e o controle de qualidade do reprocessamento desempenham papel fundamental para evitar surtos de reações pirogênicas. A literatura aponta para a ausência de procedimentos padronizados e protocolos validados nas instituições que realizam o reprocessamento de cateteres de hemodinâmica, o que pode interferir na segurança do paciente 7,14,16. Alguns autores também destacam ainda a qualidade da água utilizada no reprocessamento como um dos fatores importantes para a ocorrência de pirogenia 6,7 .

Essa recomendação está respaldada na literatura e pela legislação brasileira em vigor. Conforme a resolução da ANVISA RE no 2.606/2006 ${ }^{9}$, as instituições deverão implantar protocolos de reprocessamento que "devem garantir a qualidade do resultado e de todas as etapas do processo, incluindo a avaliação de funcionalidade, esterilidade, rastreabilidade, condições de armazenamento e descarte dos produtos". Além disso, os serviços de saúde devem promover treinamento e educação permanente da equipe e manter disponíveis os registros das reutilizações 28.

Como a probabilidade de pirogenia nos cateteres reprocessados foi a variável que mais impactou na razão de custo-efetividade incremental, o rigor no reprocessamento poderá reduzir ainda mais as chances de pirogenia, tornando a estratégia ainda mais custo-efetiva.

Em alguns países como os Estados Unidos e o Canadá, a prática do reprocessamento é comum. No primeiro, $82 \%$ das instituições reutilizam dispositivos marcados como descartáveis pelos fabricantes 16 . No Canadá, em 2001, 40\% dos hospitais com mais de 200 leitos reutiliza- vam descartáveis. Na Alemanha e na Inglaterra, o reúso também é permitido, com controle pelas autoridades. Em países como a França e a Espanha, o reúso é proibido. Em 1998, a União Europeia submeteu os reprocessadores às mesmas normas dos fabricantes 29 .

No Brasil, em 2006, a Sociedade Brasileira de Hemodinâmica e Cardiologia Intervencionista (SBHCI) reconheceu a existência da prática de reutilização de cateteres e outros materiais no Brasil, mas recomenda que a utilização dos materiais nos procedimentos diagnósticos e terapêuticos na área de atuação da cardiologia intervencionista deve obedecer às recomendações dos seus fabricantes. Caso a instituição ou profissional aja em desacordo com o fabricante, estará sujeito às responsabilidades relacionadas ao ato, mesmo diante de protocolos validados pela ANVISA. Para a SBHCI, a normatização e fiscalização dessa prática competem exclusivamente às autoridades de saúde pública, particularmente, à ANVISA 30 .

Considera-se que não há um consenso em relação a reprocessar ou não os cateteres de hemodinâmica. No entanto, a literatura aponta para a segurança do processo se o mesmo seguir protocolos rigorosos de execução.

Dentre as limitações do estudo, sinalizamos a impossibilidade de considerar, no modelo, os custos com mão de obra, tendo, em vista, a diversidade de regimes de contratação e profissionais que atuam na etapa de pré-lavagem.

O presente estudo considerou os custos praticados na instituição somados ao custo unitário do reprocessamento realizado pela empresa terceirizada. Dessa forma, os custos com equipamentos, testes, validação de processos e controle de qualidade já estão contemplados no custo unitário cobrado pela empresa terceirizada.

\section{Considerações finais}

O presente estudo não esgota todos os aspectos passíveis de discussão, inclusive por não considerar outros riscos potenciais associados ao reúso de artigos de uso único, mas consegue refletir o cenário atual da instituição e certamente contribui para a tomada de decisão dos gestores, na medida em que consegue apontar o reúso dos kits de hemodinâmica como uma estratégia de menor custo em relação ao uso exclusivo de novos. 


\section{Resumen}

El objetivo fue comparar la relación coste-eficacia en la reutilización de catéteres cardíacos respecto a los nuevos, bajo la perspectiva de un servicio público. Se utilizó un modelo analítico con el objeto de estimar la relación coste-efectividad entre las dos estrategias para el uso de materiales en el cateterismo cardíaco, utilizando la ocurrencia de reacción pirogénica como resultados clínicos. Los costes fueron estimados por la recogida directa en los sectores implicados y se expresan en reales ( $R \$)$ para el año 2012. Un diagrama de decisiones se construyó con las probabilidades pirogénicas descritas en el estudio clínico. El coste de la reutilización era de $R \$$ 109,84 y de $R \$ 283,43$ por catéteres nuevos. La estrategia de reutilización ha demostrado ser coste-efectiva y la tasa de coste-efectividad incremental indicó que para prevenir un caso pirogénico se gastarían $R \$ 13,561.75$. El estudio demuestra que la reutilización de catéteres es una estrategia de menor coste, en comparación con el uso exclusivo de los nuevos catéteres, y puede contribuir a la toma de decisiones.

Cateterismo Cardíaco; Equipo Reutilizado; Evaluación de Costo-Efectividad; Evaluación de Tecnologías de Salud

\section{Colaboradores}

B. M. G. Veras, K. M. S. Senna, M. G. Correia e M. S. Santos participaram da concepção e projeto do artigo, análise e interpretação dos dados, redação do artigo e revisão crítica relevante do conteúdo intelectual e aprovação final da versão a ser publicada.

\section{Agradecimentos}

À equipe do Núcleo de Avaliação de Tecnologias em Saúde do Instituto Nacional de Cardiologia, aos profissionais da Central de Esterilização Externa e dos demais setores administrativos da instituição.

\section{Conflito de interesses}

Não declarado.

\section{Referências}

1. Mansur AP, Favarato D. Mortalidade por doenças cardiovasculares no Brasil e na Região Metropolitana de São Paulo: atualização 2011. Arq Bras Cardiol 2012; 99:755-61.

2. Bueno MR, Ribeiro FG, Borges SR, Pavani LMD. Custo direto do reprocessamento de cateteres para estudos hemodinâmicos. Rev Soc Cardiol Estado de São Paulo 2001; 11:1-9.

3. Psaltikidis EM, Graziano KU, Frezatti F. Análise dos custos do reprocessamento de pinças de uso único utilizadas em cirurgia vídeo-assistida. Rev Latinoam Enferm 2006; 14:593-600.

4. Ultramari FT, Bueno RRL, Cunha CLP, Andrade PMP, Nercolini DC, Tarastchuk JCE, et al. Nefropatia induzida pelos meios de contraste radiológico após cateterismo cardíaco diagnóstico e terapêutico. Arq Bras Cardiol 2006; 87:378-90.

5. Cardoso CR, Prestes EP, Cardoso CO, Beulke R. Contribuição do planejamento orçamentário no gerenciamento do laboratório de hemodinâmica: simulação aplicada à gestão dos serviços de hemodinâmica. Rev Bras Cardiol Invasiva 2010; 18:62-7.
6. Duffy RE, Couto B, Pessoa JM, Starling C, Pinheiro $\mathrm{S}$, Pearson LM. Improving water quality can reduce pyrogenic reactions associated with reuse of cardiac catheters. Infect Control Hosp Epidemiol 2003; 24:955-60.

7. Ribeiro SPC. Reprocessamento de cateteres de angiografia cardiovascular após uso clínico e contaminados artificialmente: avaliação da eficácia da limpeza e esterilização [Tese de Doutorado]. São Paulo: Escola de Enfermagem, Universidade de São Paulo; 2006.

8. Agência Nacional de Vigilância Sanitária. Resolução RE no 2.605, de 11 de agosto de 2006. Estabelece a lista de produtos médicos enquadrados como de uso único proibidos de ser reprocessados. Diário Oficial da União 2006; 14 ago.

9. Agência Nacional de Vigilância Sanitária. Resolução RE no 2.606, de 11 de agosto de 2006. Produtos médicos: elaboração, validação e implantação. Dispõe sobre as diretrizes para elaboração, validação e implantação de protocolos de reprocessamento de produtos médicos e dá outras providências. Diário Oficial da União 2006; 14 ago. 
10. Scherson BA, Dighero TH. Angiographic catheter reuse at the Hemodynamic Department of a public hospital in Chile. Rev Chil Infectol 2006; 23:45-9.

11. Frank U, Herz L, Daschner FD. Infection risk of cardiac catheterization and arterial angiography with single and multiple use disposable catheters. Clin Cardiol 1988; 11:785-7.

12. Andrade MVA, Silva RS, Toni SMD, Andrade PB, Tebet MA, Labrunie A. Busca ativa de possíveis causas de pirogenia em pacientes submetidos a procedimentos coronários diagnósticos e terapêuticos. Rev Bras Cardiol Invasiva 2009; 17:234-8.

13. Jacobson JA, Schwartz CE, Marshall HW, Conti M. Burke JP. Fever, chills, and hypotension following cardiac catheterization with single- and multipleuse disposable catheters. Cather Cardiovasc Diagn 1983; 9:39-46.

14. Lucas TC, Barbosa MP, Oliveira AC. Validação do reprocessamento de cateteres cardíacos angiográficos: uma avaliação da funcionalidade e da integridade. Rev Esc Enferm USP 2010; 44:947-55.

15. Kundsin RB, Walter CW. Detection of endotoxin on sterile catheters used for cardiac catheterization. J Clin Microbiol 1980; 11:209-12.

16. Bomfim FMTS, Lima SG, Victor EG. Análise do reprocessamento de cateteres de hemodinâmica em uma capital brasileira. Rev Bras Cardiol 2013; 26:33-9.

17. Lee RV, Drabinsky M, Wolfson S, Cohen LS, Atkins E. Pyrogen reactions from cardiac catheterization. Chest 1973; 63:757-61.

18. Ajeka S, Malamam A, Silva PA. Pirogenia em cateterismo cardíaco: detecção da causa e erradicação, pela padronização do reprocessamento de materiais. Arq Bras Cardiol 2003; 81 Suppl 1:57.

19. Reyes MP, Ganguly S, Fowler M, Brown WJ, Gatmaitan BG, Friedman C, et al. Pyrogenic reactions after inadvertent infusion of endotoxins during cardiac catheterizations. Ann Intern Med 1980; 93(Part 1):32-5.

20. Cookson ST, Nora Jr. JJ, Kithas JA, Arduino MJ, Bond WW, Miller PH, et al. Pyrogenic reactions in patients undergoing cardiac catheterization associated with contaminated glass medicine cups. Cathet Cardiovasc Diagn 1997; 42:12-8.

21. Brown SA, Merritt K, Woods TO, Hitchins VM. The effects of use and simulated reuse on percutaneous transluminal coronary angioplasty balloons and catheters. Biomed Instrum Technol 2001; 35:312-22.
22. Plante S, Strauss BH, Goulet G, Watson RK, Chisholm RJ. Reuse of balloon catheters for coronary angioplasty: a potential cost-saving strategy? J Am Coll Cardiol 1994; 24:1475-81.

23. Mussivand T, Duguay DG, Valadares MJ, Rajagopalan K, Mackenzie AM, Blohon R, et al. Assessment of reused catheters. ASAIO J 1995; 41:M611-6.

24. Browne KF, Maldonado R, Telatnik M, Vlietstra RE, Brenner AS. Initial experience with reuse of coronary angioplasty catheters in the United States. J Am Coll Cardiol 1997; 30:1735-40.

25. Zubaid M, Thomas CS, Salman H, Al-Rashdan I, Hayat N, Habashi A, et al. A randomized study of the safety and efficacy of reused angioplasty balloon catheters. Indian Heart J 2001; 53:167-71.

26. Shaw JP, Eisenberg MJ, Azoulay A, Nguyen N. Reuse of catheters for percutaneous transluminal coronary angioplasty: effects on procedure time and clinical outcomes. Catheter Cardiovasc Interv 1999; 48:54-60.

27. U.S. Food and Drug Administration. Device advice: comprehensive regulatory assistance reprocessing of single-use device. http://www.fda.gov/ MedicalDevices/DeviceRegulationandGuidance/ ReprocessingofSingle-UseDevices/ucm 121067. htm (acessado em Jun/2013).

28. Agência Nacional de Vigilância Sanitária. Regras para reprocessamento de produtos médicos são atualizadas. http://www.anvisa.gov.br/ divulga/noticias/2006/210806.htm (acessado em Jun/2013)

29. Alfa MJ, Castillo J. Impact of FDA policy change on the reuse of single-use medical devices in Michigan Hospitals. Am J Infect Control 2004; 32:337-41.

30. Sociedade Brasileira de Hemodinâmica e Cardiologia Intervencionista. Novo parecer da SBHCI sobre reprocessamento de materiais em hemodinâmica e cardiologia intervencionista. http://sbhci.org.br/ profissional/notas-e-pareceres/novo-parecer-dasbhci-sobre-reprocessamento-de-materiais-emhemodinamica-e-cardiologia-intervencionista/ (acessado em Jun/2013).

Recebido em 31/Jan/2013

Versão final reapresentada em 21/Jun/2013

Aprovado em 05/Ago/2013 